\title{
Assistenza territoriale tra progettualità ed azione
}

\author{
di Elio Borgonovi
}

Una delle sfide attuali che diventerà sempre più forte nel futuro è quella del potenziamento e della valorizzazione dell' assistenza in contrasto non ospedaliero, comunemente detta territoriale. In alcune regioni essa è già sufficientemente organizzata, mentre in altre rappresenta l'anello debole se non addirittura mancante della risposta ai bisogni dei pazienti. Il sistema territoriale è costituito dalle attività legate alle cure primarie e di continuità, e da un insieme di servizi che comprende l'intera rete dei servizi sociosanitari (tipicamente $R S A, R S D, C D I, C D D, C S S, A D I$, riabilitazione, dipendenze) e alcuni servizi sanitari (ad esempio ADP, prevenzione sanitaria e veterinaria). L'offerta è garantita da una serie di operatori e fornitori complementari, rispetto ai quali però non è sempre presente una chiara azione di regia complessiva. L'evoluzione negli anni dell'offerta dei servizi è stata accompagnata da uno spostamento della spesa sanitaria verso il territorio.

Per rispondere adeguatamente alle esigenze della popolazione, varie regioni stanno perseguendo la sperimentazione di alcuni modelli innovativi di assistenza territoriale, tra cui i CReG per la presa in carico delle patologie croniche in Regione Lombardia, le case della salute in Emilia Romagna e altre.

Le sfide che tutte le regioni devono affrontare partendo da situazioni più o meno avanzate e consolidate possono essere raggruppate in tre macro-ambiti:

1) Il contesto economico ed epidemiologico.

2) La frammentazione e disomogeneità dell'attuale offerta sociale e sanitaria sul territorio.

3) L'innovazione e la ricerca di modelli assistenziali coerenti con la natura dei nuovi bisogni di salute.

Il contesto economico ed epidemiologico italiano è caratterizzato da un lato dalla "non crescita", o crescita leggera in termini nominali negli ultimi tre anni, del finanziamento (sia pubblico che privato) e dall'altro dall'aumento della popolazione cronica spesso con polipatologie, con una crescita esponenziale ripetuta per anno (ad esempio infarti, HIV, oncologici, BPCO).

Infatti, i Paesi a economia avanzata affrontano, fin dagli anni '60, una continua crescita della spesa sanitaria complessiva, pubblica e privata, dovuta a:

- Continuo sviluppo delle tecnologie sanitarie (ad esempio farmaci, vaccini, presidi, programmi di assistenza, percorsi diagnostico terapeutico assistenziali, organizzazione dei servizi) che hanno modificato l'esito di molte patologie (infettive come l'HIV, cardiovascolari, oncologiche per citarne solo tre tra le principali), e che hanno come conseguenza l'incremento dei costi di assistenza sanitaria (sopravvivenza agli eventi infausti con modifica della condizione a cronico e crescita annuale del numero, ad esempio, di infartuati sopravvissuti).

- Crescita delle aspettative di mantenimento della salute da parte della popolazione in generale, che si riverbera sulla continua richiesta di prestazioni sanitarie, e la conseguente non accettazione dello stato di "malattia" e sue conseguenze. 
- Fenomeni sociali di mobilità (tra regioni e a livello globale tra Paesi) e di condivisione comunicativa (internet) della popolazione, con l'allargamento dei confini di relazione e di informazione. Le conseguenze possono andare dalla relativa facilità di trasmissione di patologie infettive (ad esempio $\mathrm{HIV}, \mathrm{TBC}, \mathrm{HCV}$ ) fino alla conseguenza di ricerca di informazione su patologie o stati di salute percepiti attraverso percorsi liberi (false informazioni) $e$ non controllati.

Oltre a ciò l'Italia presenta due ulteriori elementi di criticità:

- Aumento della popolazione anziana, come conseguenza dello sviluppo delle conoscenze e delle tecnologie e quindi della sopravvivenza agli eventi infausti: l'Italia sta diventando un Paese anziano, tanto da contendere al Giappone il primato mondiale del più alto indice di vecchiaia e, come è noto, gli assistiti anziani costano di più (soprattutto negli ultimi due anni di vita) e richiedono un'assistenza sanitaria distribuita sul territorio con prossimità al loro domicilio.

- Pressione economica e relative tensioni finanziarie che negli ultimi anni stanno condizionando il Paese. La previsione a medio periodo è, di fatto, un'invarianza di finanziamento, in presenza di una spesa che tendenzialmente creerà alle regioni problemi di deficit nei loro bilanci.

Diventa quindi importante sviluppare e implementare modelli e strumenti che consentano lo spostamento dell' asse di cura verso il territorio in modo efficace dal punto di vista clinico e socioassistenziale ed economicamente sostenibile. Di fronte a queste tendenze strutturali si rileva che oggi l'offerta territoriale sociale e sanitaria, anche nelle regioni dove essa è più avanzata, risulta costituita da un universo di produttori e di attori, che presenta alcune potenziali aree di miglioramento. In particolare:

- Non è sempre rintracciabile una chiara azione di regia complessiva, i percorsi e le opzioni sono difficilmente riconoscibili da cittadini e operatori stessi e non sono omogenei sul territorio regionale.

- In generale l'offerta non risponde in modo integrato ai bisogni complessivi dei pazienti (sia di tipo sociale che sanitario) ed è causa di sovrapposizioni e non chiarezza, cui si aggiunge la non ottimizzazione della qualità dell'assistenza.

- La discontinuità tra ospedale e territorio e tra diversi operatori del territorio stesso nasce anche da un rapporto e confronto tra professionisti non sempre ideale e collaborativo, ma più spesso inesistente/minimale e/o conflittuale.

- Mancano o vengono scarsamente utilizzati e diffusi strumenti di misura e valutazione dell'efficacia terapeuticoassistenziale ed economica.

Il superamento della frammentazione e disomogeneità dell'attuale offerta sociale e sanitaria sul territorio è condizione necessaria per garantire ai cittadini una presa in carico reale e continuativa, in tutte le fasi e momenti del proprio percorso diagnostico-assistenziale-terapeutico, in ambito socio-sanitario-assistenziale. Si tratta di una sfida non facile da vincere in quanto sistemi sanitari sono oggi caratterizzati da una grande complessità dovuta a:

- Innumerevoli tecnologie sanitarie che vengono utilizzate (farmaci, vaccini, programmi di assistenza, PDT/ PDTA, modelli organizzativi).

- Dinamicità del contesto che richiede da parte degli operatori un continuo adattamento al variare delle conoscenze scientifiche e delle esigenze organizzative.

- Mutata relazione medico-paziente, con quest'ultimo sempre più informato ed esigente.

- Mutato atteggiamento della società nei confronti della medicina, con aspettative dei pazienti a volte superiori a quanto la medicina può fare e che sconfinano in quelle che si possono definire "pretese" e non esercizio di diritti. 
Mentre l'assistenza per acuti, tipicamente in ospedale, può fare riferimento a modelli di ricerca e di formazione consolidati, anche se strutturalmente in ritardo in un mondo che cambia rapidamente, lo sviluppo dell'assistenza territoriale richiede una forte innovazione nella ricerca e nei modelli formativi. Il modello attuale di formazione continua non sfrutta il potenziale di impatto formativo della ricerca scientifica. Questa infatti consente di capire il rapporto tra teoria e pratica, di portare il proprio contributo, di partecipare alla preparazione, alla conduzione e alla raccolta dei risultati, allena a diventare più critici ad interpretare il lavoro degli altri, a discriminare tra risultati utili e futili. In tal senso la ricerca scientifica dovrebbe essere estesa a tutto il personale del SSN e in particolare ai MMG, ai PLS, ai medici, infermieri e altri operatori socio-sanitari e assistenziali operanti sul territorio.

Peraltro, oggi la responsabilità della formazione dei professionisti che operano sul territorio è delegata alle Regioni che hanno sviluppato modelli ed esperienze differenti, il che è stato un ulteriore elemento di divaricazione in quanto l'assistenza in contesto non ospedaliero risente maggiormente delle condizioni socio-economiche e dei contesti delle diverse comunità e territori.

La ricerca e la formazione devono essere guidati da una visione e da strategie di lungo periodo che dovrebbero fare riferimento alle seguenti linee di indirizzo. Innanzitutto la logica della presa in carico "globale" dell'individuo e della famiglia, attraverso un modello che superi l'attuale frammentazione del sistema, persegua nei fatti l'integrazione socio-sanitaria-assistenziale e garantisca la continuità all'interno del percorso di cura/assistenza tra $i$ diversi attori e fornitori di servizi. Gli elementi fondamentali del modello di integrazione socio-sanitariaassistenziale sono:

- La valutazione multidimensionale del bisogno, individuando i diversi gradi di complessità della condizione del paziente, dal punto di vista assistenziale, sanitario, socio-relazionale ed ambientale. La Valutazione Multidimensionale si avvale di scale validate e condivise ed è effettuata da alcune figure stabili (MMG, infermiere e assistente sociale) e altre figure specialistiche che intervengono "secondo necessità" (medici specialisti, fisioterapista, educatore).

- L'unitarietà dell'intervento e del percorso assistenziale, con concorso professionale di personale medico, infermieristico, riabilitativo e di servizio sociale professionale.

- Il progetto personalizzato che tenga conto degli obiettivi di salute da raggiungere, del responsabile del caso (case manager) e della verifica delle azioni compiute sotto il profilo della qualità e degli esiti.

In secondo luogo, la presa in carico "attiva" della fragilità e della cronicità, che consenta di gestirle, anche prima del verificarsi degli episodi di acuzie, in percorsi socio-sanitari-assistenziali definiti e monitorati. Concetti delineati all'interno del Chronic Care Model (o nella sua evoluzione Expanded Chronic Care Model), che infatti si basa sulla capacità del paziente di intervenire sul proprio stato di salute insieme al lavoro integrato di diversi. Il modello si basa sui seguenti principi:

- Organizzazione multidimensionale e di team dell'assistenza, che affronti in modo integrato ed "olistico" il bisogno di salute degli individui.

- Proattività degli interventi, le consuete attività cliniche e assistenziali sono integrate e rafforzate da interventi programmati di promozione della salute, educazione sanitaria (anche a famiglie e caregiver), prevenzione, follow-up con sistemi automatici di allerta e di richiamo.

- Supporto a self management e empowerment del paziente, con l'obiettivo di aiutare i pazienti e le loro famiglie ad acquisire conoscenze, abilità e motivazioni nella gestione della malattia, procurando gli strumenti necessari e valutando regolarmente i risultati e i problemi.

- Adozione di linee guida, percorsi diagnostici terapeutici assistenziali e protocolli basati sull'EBM, condivisi in riunioni periodiche del team.

- Sistemi informativi a supporto del team, ad esempio attraverso: funzioni di allerta, monitoraggio dei livelli di 
performance e aderenza alle linee guida, registro di patologia, monitoraggio e valutazione dei progetti e degli interventi.

- Sviluppo di condizioni sociali favorevoli, attraverso l'alleanza con le associazioni di cittadini e gruppi di volontariato, lo sviluppo di gruppi di auto-mutuo aiuto.

In terzo luogo, la prossimità e facilità di accesso ai servizi, garantendo che i cittadini, anche quelli che abitano lontano dai centri principali possano trovare una "porta di accesso" adeguata al sistema (importante soprattutto in caso di riorganizzazione dei presidi ospedalieri), la semplificazione dell'accesso (anche sburocratizzazione) e riconoscibilità dei servizi, non solo sanitari e sociali, ma anche amministrativi di cui i cittadini (anche sani) devono usufruire (ad esempio cambio medico, certificato). Si può sottolineare che le tecnologie di comunicazione a distanza e adeguate azioni di informazione ai cittadini sono indispensabili.

In quarto luogo, occorre definire e applicare specifici sistemi di misurazione dell'efficacia terapeutico assistenziale ed economica dei percorsi individuati, delle prestazioni erogate e dei diversi operatori ed attori, con l'obiettivo di indirizzare e perseguire il più appropriato utilizzo delle risorse pubbliche e la sostenibilità del sistema stesso. Esistono molti e sofisticati sistemi di misurazione delle performance per quanto riguarda l'assistenza ospedaliere (ad esempio piano nazionale esiti, indicatori proposti da Agenas, indicatori definiti dalle aziende in applicazione dei sistemi di remunerazione dei risultati), mentre sono meno numerosi, meno omogenei, meno condivisi e più complessi gli indicatori applicabili all' assistenza territoriale che ha gradi di multidimensionalità più elevati rispetto all' assistenza per acuti. Ultima, ma non certo per importanza, va ricordata l'integrazione ospedale e territorio (con tutti i molteplici operatori e fornitori di servizi) per una efficace presa in carico e accompagnamento senza frammentazione del percorso di cura. La logica dell'assistenza per acuti è quella di portare i pazienti verso i luoghi più appropriati di cura (ad esempio distinguendo nel modello hub \& spoke il ruolo dei diversi presidi), quella dell' assistenza territoriale è esattamente opposta, in quanto significa portare i servizi e le risposte verso i cittadini e i luoghi nei quali essi vivono.

Sul tema dell'assistenza territoriale sono state fatte tante analisi e formulate proposte di carattere generale, molte volte generiche. Si è adottata spesso la logica dei modelli ottimali, dei riferimenti delle "best (o good) practices" non di rado mutuate dall'estero, in ragione della esterofilia che ha caratterizzato il nostro Paese prima dell'avvento del "vento sovranista" che sta soffiando in Europa e nel mondo occidentale. Proposte di soluzioni ottimali che in gran parte sono state carenti dall'analisi di fattibilità. Per cercare di colmare in parte questa carenza si può tentare di definire alcuni interventi collegati alle diverse linee di indirizzo.

Per la prima linea si può proporre la costituzione di Centri sociosanitari territoriali (in alcune regioni già esistono con diverse denominazioni e con diversi livelli di funzionamento, in altre sono completamente assenti). Sono pensati come il punto di accesso riconoscibile al sistema della salute. Rappresentano il luogo di incontro tra le diverse figure professionali e un elemento di integrazione tra l'ospedale e il territorio. Come tali devono essere caratterizzati da tempi di apertura compatibili con i ritmi della vita lavorativa e aperti alle esigenze di una società multiculturale: luoghi di ascolto, accompagnamento e presa in carico; luoghi di salute, non ospedali, non pronti soccorso e neppure semplici uffici amministrativi. Centri che dovranno garantire:

- L'organizzazione della presa in carico dei cittadini attraverso sistemi di valutazione multidimensionale del bisogno standardizzati a livello regionale.

- Un'attività di indirizzo verso i diversi nodi della rete a seconda delle esigenze rilevate, anche in accordo e in collaborazione con i Comuni per quanto di loro competenza.

- L'erogazione di alcune prestazioni sanitarie, quali ad esempio medicina generale, assistenza infermieristica, prelievi per analisi di laboratorio, diagnostica strumentale semplice (ECG, Eco, Spirometro, Saturimetro) ma anche prevenzione su group risk adjusted, visite specialistiche, formazione per $i$ care givers.

- L'apertura 8-20 agli assistiti nei giorni feriali nonché nei giorni prefestivi e festivi con idonea turnazione, anche per prestazioni di primo soccorso secondo quanto previsto dalla L. 189/12. 
- Lo sviluppo di una sanità di iniziativa, attraverso campagne di prevenzione, educazione sanitaria e attività fisica adattata.

- La presenza "concentrata" di un'offerta di servizi socio sanitari oggi dispersi sul territorio e poco conosciuti, quali ad esempio quelli erogati dai consultori, dai centri vaccinali o dai centri diurni.

- La possibilità di ospitare attività di terzo settore funzionali alla risposta dei bisogni di salute.

Il Centro Sociosanitario, dovendo garantire una presa in carico e un accompagnamento multidimensionale, dovrebbe prevedere la presenza delle seguenti figure:

- MMG e PLS in forma organizzata.

- Figure infermieristiche, anche con l'obiettivo di sperimentare ruoli di Care Manager/Infermiere Tutor.

- Disability and Case Manager, come indicato dal recente Piano Nazionale Disabilità.

- Educatori/psicologi/assistenti sociali/operatori del settore.

- Medici specialisti per le patologie a maggior diffusione e la possibilità di attivare second opinion specialistiche a distanza grazie all'uso di strumenti di telemedicina.

- Associazioni di volontariato.

La progettazione di queste realtà territoriali dovrà tener conto della localizzazione e del contesto di relazioni in essere nella specifica ASL. Sarà necessario immaginare configurazioni diverse a seconda che il Centro sia localizzato in una città metropolitana, in una piccola città di provincia, in una zona montana e/o rurale, dove è impensabile sguarnire completamente i piccoli paesi dall'assistenza del MMG. Analogamente la progettazione dovrebbe cogliere le opportunità offerte dallo stato delle relazioni tra i diversi attori nelle varie ASL. In alcuni territori infatti le ASL hanno nel tempo costruito relazioni di valore con MMG/PLS e sperimentato modelli di presa in carico della cronicità e della fragilità che è importante valorizzare.

In questo senso, qualora il contesto lo renda opportuno, il centro sociosanitario territoriale potrebbe prevedere anche la possibilità di ampliare il perimetro dell'offerta. Ad esempio potrebbe:

- Effettuare prestazioni di ambulatorio specialistico, nel rispetto della programmazione della ASL.

- Effettuare diagnostica per immagini anche con metodiche radiologiche, in coerenza con il piano territoriale.

- Effettuare prestazioni riabilitative (con particolare riferimento alle ambulatoriali) coerentemente al disegno di riordino in atto.

- Ospitare servizi per l'area della salute mentale, coerentemente al disegno che si verrà a delineare in ogni regione.

- Organizzare la continuità assistenziale sostituendo la guardia medica per i propri assistiti.

- Effettuare prestazioni di urgenza per i propri assistiti (presa in carico durante l'apertura dal primo medico disponibile).

- Coordinare ADI e ADP.

- Astanteria ove l'organizzazione e la struttura fisica lo permettano.

Elemento necessario del Centro Sociosanitario Territoriale è un sistema informativo - integrato con il SISS - in grado, tra le altre cose, di mappare la domanda di servizi e il tipo di risposta fornita, di gestire le scale di valutazione multidimensionale in uso, la predisposizione e aggiornamento di piani terapeutici/percorsi individuali e di raccogliere indicatori clinici e di esito sui diversi percorsi attivati. Questa condizione permetterà al centro di avviare l'attività di ricerca, valutazione e governo delle attività che caratterizzerà il prossimo futuro dell'assistenza. La Governance del Centro Sociosanitario Territoriale dovrà essere definita dalla Regione in rapporto al proprio modello istituzionale, in modo da garantire le attività di analisi del bisogno, indirizzo e regolamentazione, programmazione, 
contrattualizzazione e controllo dei servizi che verranno erogati nel Centro dai possibili diversi fornitori (ad esempio gruppi di medici di famiglia, assistenti sociali, infermieri, etc.). La gestione dei servizi potrà essere realizzata in forme differenti (cooperative, società di servizi, etc.). Ovvero, i diversi servizi, sotto la responsabilità di diversi attori e istituzioni saranno localizzati in spazi fisici contigui, utilizzando sistemi informativi compatibili (che diventano elemento di monitoraggio, scambio informativo e controllo) e condividendo momenti di programmazione congiunta sulla base di obiettivi condivisi e dei bisogni dell'utenza.

Le nuove sfide che il Sistema Sanitario Nazionale deve affrontare richiedono un ripensamento dei ruoli storicamente giocati dai diversi professionisti in modo da valorizzare le professionalità e le competenze delle diverse figure professionali. Il MMG oltre alle attuali attività di cura può essere uno dei registi delle attività di presa in carico, attivando figure professionali e strumenti (e.g. diagnostica, unità d'offerta sociosanitaria) in un nuovo ruolo di coordinamento e governo clinico, superando la presa in carico individuale dell' assistito e mettendo in pratica un modello di assisten$z a$ di team e multidimensionale. La figura dell' infermiere evolve nella direzione di Care Manager, ovvero identifica le esigenze assistenziali di tipo infermieristico (assistenza domiciliare, controllo dei parametri, educazione sanitaria) e ne coordina l'erogazione. Lo specialista ospedaliero è di volta in volta coinvolto dal MMG a seconda della specificità del bisogno complesso (ad esempio doppia diagnosi, dipendenze, ... ) e deve consentire il raccordo e la continuità con il presidio ospedaliero di riferimento (per territorio e patologia). Le altre figure professionali dell'assistenza sociale identificano le esigenze di tipo socio-assistenziali e seguono gli interventi, confrontandosi e osservando le linee guida condivise con le istituzioni di riferimento.

Le diverse forme di integrazione oggi presenti o in sperimentazione andrebbero messe a sistema e fatte evolvere secondo un disegno complessivo e coerente, che realizzi un modello di presa in carico proattiva, integrata e multidimensionale. In particolare per quanto riguarda i modelli di sperimentazione da alcuni anni varie regioni di presa in carico dei pazienti cronici convergono su questi interventi:

- Intervenire in modo proattivo sui pazienti fragili, introducendo accanto alla dimensione clinica la dimensione sociale.

- Intervenire con azioni di promozione, prevenzione e di educazione sul paziente, sulla famiglia e sul caregiver, anche attraverso le figure infermieristiche ed assistenziali.

- Definire, monitorare e valutare obiettivi di risultati di efficacia terapeutica (sviluppo ed evoluzione del PAI e adesione ai PDTA, adesione alla terapia, riduzione delle ospedalizzazioni, etc.), e di costo (prescrizioni diagnostiche, farmaceutiche, etc.).

A supporto dell'obiettivo di continuità assistenziale andranno sviluppati strumenti e modelli di relazione che consentano al Centro Socio-sanitario Territoriale, al Presidio Ospedaliero Territoriale (ospedali di prossimità o altre definizioni simili) e agli altri operatori sul territorio (RSA, cure intermedie, riabilitazione) di formare un sistema di offerta integrato. Andrà garantita in particolare la continuità assistenziale non solo nei momenti di diagnosi e cura, ma soprattutto nelle discontinuità (ad esempio ricovero e dimissioni). I modelli di collaborazione dovranno far leva sulla collaborazione tra i diversi professionisti, la valorizzazione delle sinergie e ottimizzazione dei percorsi, le cartelle cliniche e PAI condivisi.

Formazione e Ricerca sono fondamentali per migliorare le competenze degli operatori sanitari e per partecipare in modo attivo all'espansione delle conoscenze. Le due funzioni sono interattive e idealmente il modo migliore per realizzare la formazione è la partecipazione a progetti di ricerca, trasformando il Servizio Sanitario in un grande laboratorio dove idee e azioni ruotano attorno alla centralità del cittadino e dell'ammalato. Si intende in questo modo utilizzare i mezzi informativi messi a disposizione attraverso le varie banche dati esistenti. Lo spettro della ricerca dovrà essere il più vasto possibile, dovrà coinvolgere tutti gli operatori delle regioni. Si tratta di sviluppare una ricerca epidemiologica con nuove metodologie sostenute soprattutto dall'enorme mole di dati oggi disponibili e facilmente accessibili. I cosiddetti big data consentiranno se ben utilizzati di applicare una metodologia di real data 
evidence, cioè evidenze non emerse da sperimentazioni di laboratorio trial clinici, ma dagli effetti sullo stato di salute di trattamenti che usano una molteplicità di farmaci ed altri trattamenti socio-sanitari e assistenziali.

Questo implica lo sviluppo di un programma pluriennale di ricerca, finanziato in modo adeguato, secondo alcune direttrici che dovranno essere coordinate a livello interregionale per evitare duplicazioni e favorire le collaborazioni. La formazione, che ovviamente dovrà coinvolgere le università e centri post-universitari, dovrà anche comprendere elementi che favoriscano il superamento delle barriere (anche culturali) tra ospedale e territorio, favorendone l'integrazione, ad esempio facendo conoscere a tutti gli aspetti salienti dell'uno e dell'altro, sviluppando tirocini anche sul territorio.

Il rafforzamento del sistema di monitoraggio e valutazione e l'evoluzione del sistema di incentivi sono un elemento chiave per lo sviluppo sostenibile e di qualità dell'assistenza territoriale. Questo implica tre linee di intervento: flussi informativi ricchi e tempestivi, momenti di confronto sugli outcome/esiti di salute, sviluppo diforme di incentivazione finalizzati e specifici.

Le politiche pubbliche italiane sono caratterizzate da due grandi punti di debolezza: difficoltà e ritardi nell'attuazione/implementazione e monitoraggio dei risultati. Usando una frase ad effetto si potrebbe dire che moltissimi sono bravi (o hanno imparato) a fare le analisi su ciò che va e ciò che non va, molti (compresi accademici e consulenti) sono bravi nel dire "cosa si dovrebbe fare", un numero minore è in grado di governare i processi politici in modo da arrivare alle decisioni (ad esempio approvare leggi o decreti sul potenziamento dell'assistenza territoriale), un numero assai più basso sa ò è disposto a imparare come si possono applicare le politiche, un numero ridottissimo è quello che si impegna realmente a fare, pochi se non nessuno si preoccupano di verificare come sono andate veramente le cose per intervenire con la logica di "cambiare le cose che non vanno senza buttar via quelle che vanno", ossia "buttar via l'acqua sporca senza buttar via il bambino". La sfida per l'assistenza territoriale è quella del potenziamento e innovazione, dove già esiste, consolidamento, dove è stato introdotto da poco, introduzione, dove non esiste o è appena accennato. Anche se potrà fare affidamento su nuove tecnologie, soprattutto ITC, occorre la consapevolezza che "tra il dire e il fare c'è di mezzo il mare", ma che per navigare bene in mare occorre avere una bussola, un orientamento e occorre saper affrontare le situazioni e le condizioni che si creano giorno per giorno, ossia saper affrontare $i$ dettagli. 